\title{
Measuring Adolescent Chronic Stress: A Review of Established Biomarkers and Psychometric Instruments
}

\author{
Ioulia Kokka ${ }^{a, b}$ George P. Chrousos ${ }^{a, c}$ Christina Darviri ${ }^{a}$ Flora Bacopoulou $^{a, c, d}$ \\ aPostgraduate Course on the Science of Stress and Health Promotion, School of Medicine, National and Kapodistrian \\ University of Athens, Athens, Greece; ${ }^{\text {b}}$ First Department of Psychiatry, Outpatient Specialty Clinic for Obsessive \\ Compulsive Disorder and Behavioral Therapy, Eginition Hospital, National and Kapodistrian University of Athens, \\ Athens, Greece; 'University Research Institute of Maternal and Child Health \& Precision Medicine and UNESCO Chair \\ in Adolescent Health Care, Aghia Sophia Children's Hospital, National and Kapodistrian University of Athens, \\ Athens, Greece; ${ }^{\text {d}}$ First Department of Pediatrics, Center for Adolescent Medicine and UNESCO Chair in Adolescent \\ Health Care, School of Medicine, Aghia Sophia Children's Hospital, National and Kapodistrian University of Athens, \\ Athens, Greece
}

\section{Keywords}

Adolescence · Stress · Psychometric instruments ·

Questionnaire · Biomarkers

\begin{abstract}
Adolescence is a period of stressful physiological and psychosocial changes. Exposure to chronic stress can cause specific structural and functional changes in an organism, which can be appraised objectively. Some of these alterations are an expected reaction of the body in its attempt to adapt to a stressful situation, while others are signs of possible disease development. The aim of this review was to present the most widely used methods of stress evaluation in adolescence research. Primary biomarkers associated with different biological systems, such as the stress hormones glucocorticoids, and catecholamines, as well as the available methods of extraction and assessment of each biomarker, are presented. This work also includes secondary outcomes, which can also provide an estimation of an individual's stress level. Also, most available psychometric instruments of stress, constructed to address specifically this period of life, are presented and discussed. In addition, this paper addresses possible confounding factors that may affect stress mea-
\end{abstract}

surements, which should be taken under consideration when conducting stress research. To objectively evaluate stress, it is of great importance for a researcher to be familiar with the condition under examination and its representative stress indices. Adequate evaluation of adolescents with the selection of proper psychometric tests and biological markers can help design targeted interventions aiming to prevent or reverse the effects of physical and mental stressors that occur during adolescence, effects that can be carried into adulthood with detrimental consequences.

(c) 2022 S. Karger AG, Base

\section{Introduction}

The somatic, psychological, and social changes and adjustments that occur during adolescence render this period of life quite stressful for an individual [1]. Indeed, adolescence is one of the most stressful and stress-sensitive developmental stages of life, during which long-term stress exposure may lead to chronic dysregulation of both physical and mental homeostasis [2], with possible detrimental consequences that can be carried into adulthood [3]. Understanding the magnitude of chronic stress ef- 
fects on adolescents is of great importance in terms of health promotion, prevention, and restoration. For this paper's purposes, chronic stress is defined as the exposure to stressful stimuli which, when perceived as unsurpassed, results in protracted stress hormone release (primary mediators), which leads to adverse secondary outcomes. The aim of this paper was to present those primary biomarkers and secondary outcomes which, when measured, provide a clear perspective of the impact of stress on adolescents. Available psychometric instruments addressing the same topic with respect to adolescence are also presented. The present work constitutes a narrative review, aiming at a qualitative, rather than a systematic, approach to the existing literature on chronic adolescent stress evaluation methods. This paper does not seek to generalize cumulative information, but to synthesize knowledge on adolescent stress biomarkers and its psychometric instruments. Although this unstructured approach bears the limitation of lacking explicit criteria [4], it may aspire research ideas by highlighting the strengths and weaknesses of adolescent stress measurement methods and, thereby, assist in formulating research questions and hypotheses [5].

\section{Human Stress Response}

According to Lazarus's theory on the cognitive process of stress, this includes three components: the stressful stimulus (external component), the appraisal of the stressor (internal component), and the stress response (combination of the components) [6]. When examining stress, two clarifications need to be made. The first one is the differentiation of stress exposure and stress response; while stress exposure refers to those events that could potentially alter the typical biopsychological function, stress response refers to the emotional, cognitive-behavioral, and biological responses to stress exposure, depending on the coping skills of the individual [7]. Thereby, stress response is the outcome of the individual's evaluation of the stressor. Secondly, stress can be categorized as beneficial (eustress) or harmful (distress) depending on the way the individual perceives a stressor; eustress refers to the positively focused energy to successfully manage a stressful experience, whereas distress refers to the maladaptive behavior and unsuccessful management of that experience [8]. The key element of the cognitive-behavioral and physiological adaptation to a stressful stimulus, the socalled fight-or-flight response, is the activation of the autonomic nervous system as well as of the hypothalamic- pituitary-adrenal axis (HPA). The fight-or-flight response was introduced by Cannon in the mid-1920s to describe the animal response to danger. According to this, if the danger is managed properly, the body reachieves homeostasis through the activation of the "relaxation response"; decrease in the heart rate, secretion of stress hormones, and deceleration of breathing [9]. When an individual faces multiple unrelenting stressors or a single, persistent stressor, the relaxation response cannot be activated. Chronic exposure to stress leads to persistent stress hormones' release, which negatively affects brain development during adolescence [10] and disrupts related homeostatic systems such as the endocrine, cardiorespiratory, and immune systems [11]. Such disruptions, perceived as secondary outcomes of the stress response, may lead to disease that can be carried into adulthood and thus affect the entire lifespan [12]. This "path" followed by an organism under stress is not linear; on the contrary, it is a complex sequence affected by the individual's personality traits and behavioral patterns, as well as by environmental factors (Fig. 1).

There are two different approaches to evaluate stress levels and its impact during adolescence, the psychometric, self-report instruments, and specific biomarkers. The latter are used as indicators of altered biological functions and are of great diagnostic and prognostic importance. Stress biomarkers can be classified as neuroendocrine and immune.

\section{Primary Biomarkers of Chronic Stress}

\section{Neuroendocrine Biomarkers}

Neuroendocrine biomarkers of chronic stress are effective stress indicators as the neuroendocrine system is the first one to react to a stressful stimulus and affects the response of systems to the same stimulus, including the cardiovascular and the immune system. Simultaneously, it has a role in energy production and behavioral adaptation, therefore, assisting in re-achieving homeostasis [13]. Neuroendocrine biomarkers include the glucocorticoid cortisol, the catecholamines (adrenaline, noradrenaline, dopamine), and dehydroepiandrosterone (DHEA).

Cortisol is a glucocorticoid, end-product of the HPA axis. Measurement of cortisol is feasible in serum, saliva, urine, hair, and recently in nails. Serum cortisol has been used for the evaluation of the adolescent acute stress response; yet is not widely used for the measurement of chronic stress [14]. Salivary cortisol has been the most common biomarker measured during adolescence for 
Fig. 1. The biological response to chronic stress. When the individual identifies a stressor or perceives a situation as stressful, primary stress biomarkers are activated. Protracted secretion of those results in rupture of various systems, which in their turn can lead to chronic illness.

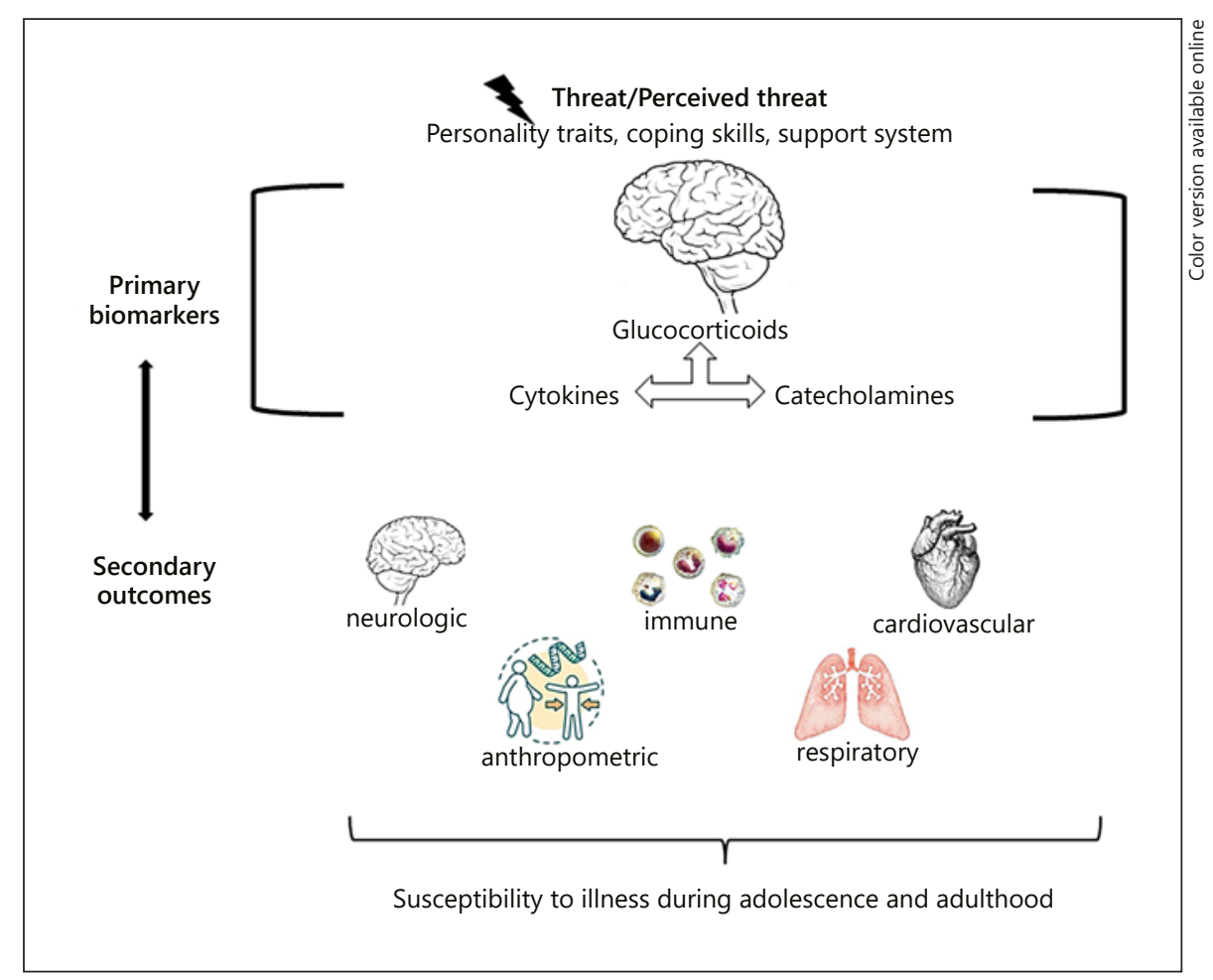

more than 20 years, as it is noninvasive and enhances participants' compliance [15-17]. Due to the HPA axis's diurnal rhythm, cortisol is best measured with multiple samplings throughout a day. Most research protocols of diurnal measurements include the cortisol awakening response 30-45 min post-awakening; the diurnal cortisol slope, which reports the cortisol change from morning to evening; and the area under the curve, which reports all cortisol data throughout the day [18]. Elevated saliva cortisol during adolescence has been linked to mental health conditions such as depression, phobias, bullying, family conflict, and obesity [19-23]. Unbound urinary cortisol can be characterized as much more efficient than serum or salivary cortisol, as it provides a 24-h clear image of adrenocortical activity. This method of measurement is typically used in clinical practice to diagnose hypercortisolism in medical conditions such as Cushing syndrome more frequently when compared to serum or saliva cortisol [24-26]. However, this method of 24-h urinary-free cortisol measurements, due to its time integrated nature, is unfit to study the responsivity of the HPA axis to a stressor.

For more than a decade, researchers have been measuring cortisol levels and the effect of chronic stress in various age-groups and conditions, using hair samples
[27]; this method is considered superior compared with the serum and urine measurements, as it facilitates evaluation of cortisol over time, with each centimeter of extracted hair representing the cortisol level of the previous month. Studies have associated hair cortisol concentration with psychopathology, traumatic experiences, obesity, and other physical illnesses [28-31]. The newest, alternative method of cortisol assessment is that obtained from extraction from the nails; preliminary evidence has shown that nail cortisol is associated with higher risk for acute coronary syndrome and depression [32]. Literature search for the purposes of this paper retrieved only 1 study on adolescents which examined the association of nail cortisol and stressful events and sleep disturbances and revealed a significant correlation among them [33].

Catecholamine (adrenaline, noradrenaline, and dopamine) release is provoked under a threat or perceived threat. Similarly to glucocorticoids, catecholamines are related to metabolic, cardiovascular, and immune functions and can provide an estimate of stress [34].

Adrenaline, produced by the adrenal medulla, and noradrenaline, produced mostly by sympathetic neurons, are parts of the sympathetic nervous system's reaction to a stressor. Adrenaline is primarily linked to emotional distress, while noradrenaline is mostly linked to the phys- 
ical activation [35]. Although they are distinct indices, they are usually measured together as a chronic stress index and have been tested in relation to adolescent anxiety, obesity, and several other physical illnesses [36, 37]. Both adrenaline and noradrenaline can be measured in urine and blood serum, however, researchers prefer the $24-\mathrm{h}$ catecholamine urine testing, as serum measurements can give false-positive results due to the stress induced by venipuncture or false-negative results due to rapid local catabolism.

Dopamine is a neurotransmitter, constituting more than $80 \%$ of the catecholamines in the brain. It has a fundamental role in the function of the hedonic reward system and affects the decision-making and behavioral reactions to stress-inducing events [38]. Due to its relation to the ongoing process of cognitive maturation during adolescence, the importance of dopamine increase is unique; it determines the positive or negative response to external stressful influences [39] and is linked to an elevated risk for the development of mental disorders [40]. As with adrenaline and noradrenaline, dopamine can be measured in serum or 24 -h urine samples.

DHEA is one of the effluent steroid hormones produced by the adrenal glands. It suppresses pro-inflammatory cytokines and enhances lipid metabolism [41]. DHEA can be measured in serum, saliva, and urine [42]. In serum, DHEA is usually measured in its sulfated, inactive form (DHEA-S). Both forms have been found to reach their peak during the morning hours. It has been shown that, when examining chronic stress in adults, the lower the DHEA, the more active the HPA axis is [43]. Interestingly, this does not seem to apply in adolescents; in a study examining the association between DHEA and adolescent stage, increased cortisol was associated with increased DHEA, but this relation was reversed only for those who had early life stressful experiences [44]. Another study, which evaluated the cortisol-DHEA relation in adolescents with specific stressful experiences, showed that the two hormones had the same rhythm of secretion [45].

\section{Immunological Biomarkers}

Cytokines are protein hormones, which contribute to the communication between cells. Cytokines can have either an autocrine, paracrine, or endocrine action and are categorized as pro- or anti-inflammatory [46]. Cytokines function as part of a bidirectional procedure between the immune and the nervous system; when the individual is under stress, catecholamines provoke the release of inflammatory cytokines, which contribute to the activation of the HPA axis and the secretion of cortisol, which then inhibits pro-inflammatory cytokines. When stress exposure is chronic, this procedure is obstructed and can lead to deficient immune response [47]. Interleukin (IL)-6, IL$1 \beta$, tumor necrosis factor-alpha (TNF- $\alpha$ ), C-reactive protein (CRP) are some of the immune factors currently in use as biomarkers for chronic stress. Insulin-like growth factor-1 (IGF-1), a hormone secreted by the liver and other cell types and can be measured in serum, has been used as an index of chronic stress.

IL-6 circulating in the bloodstream is elevated under chronic stress; long-term exposure to stress-inducing events may reprogram the immune response of an organism toward its chronic activation [48]. Following this activation, high levels of IL-6 during adolescence may lead to physical illnesses in adulthood, such as metabolic syndrome and cardiovascular disease [49]. Research on IL-6 in adolescent populations has shown that high levels of this cytokine have been related to psychosocial and environmental factors; the absence of a supportive social system, poor socioeconomic status, and sexual abuse have been linked to elevated IL-6 [50].

IL- $1 \beta$ is produced by activated macrophages and other immune cells and is one of the most important mediators of the inflammatory reaction as it is related to any autoimmune inflammation [51]. Although other cytokines are also important for the stress system, IL- $1 \beta$ was the first one to be associated with the HPA axis and the stress response [52]; it provokes the secretion of the corticotropin-releasing hormone, which stimulates the secretion of the adrenocorticotropic hormone, which in turn enhances the secretion of glucocorticoids [53]. With respect to adolescence, IL- $1 \beta$ research so far has mainly focused on overweight populations, where increased fat percentage has been associated with higher levels of IL-1 $\beta$ [54].

TNF- $\alpha$ is a multifunctional cytokine that is involved in many different pathways regarding the homeostasis and pathophysiology of mammals. It is involved in various biological actions of the metabolic and immune systems and has a complex regulatory function [55]. In preadolescents, elevated plasma TNF- $\alpha$ levels have been associated with stressful life events [56].

CRP is a protein circulating in blood; it is part of the acute phase reaction and increases when inflammation is present [57]. Therefore, it can be a useful biomarker of inflammatory conditions. It is known that chronic stress increases the secretion of IL- $1 \beta$ and IL- 6 and provokes a low-grade inflammation, thus, CRP can be used to screen for the presence of a possible inflammatory response to chronic stressors. A study of adolescents under chroni- 
Table 1. Primary biomarkers of chronic stress, biological tissues in which they are measured, and main stress-related functions

\begin{tabular}{|c|c|c|}
\hline Cortisol & Serum, saliva, hair, nails, CSF & Regulates/supports important cardiovascular, metabolic, immunologic, and other homeostatic functions \\
\hline Adrenaline & Serum, urine & $\begin{array}{l}\text { Has important roles in the fight-or-flight response by increasing blood flow to brain, heart, and skeletal } \\
\text { muscles }\end{array}$ \\
\hline Noradrenaline & Serum, urine, CSF & Mobilizes cardiometabolic resources to assist homeostasis \\
\hline Dopamine & Serum, urine, CSF & Associated with cognitive-behavioral and cardiovascular stress responses \\
\hline DHEA & Serum, saliva, urine & Suppresses pro-inflammatory cytokines and enhances lipid metabolism \\
\hline TNF- $a$ & Serum & Stimulates inflammation; produced locally by immune cells \\
\hline CRP & Serum & Associated with inflammatory reaction \\
\hline IGF-1 & Serum & Important for growth, anabolic function \\
\hline
\end{tabular}

CRP, C-reactive protein; CSF, cerebrospinal fluid; DHEA, dehydroepiandrosterone; IGF-1, insulin-like growth factor-1; IL, interleukin; TNF-a, tumor necrosis factor-a.

cally stressful experiences revealed circulating CRP levels above the cutoff values [58]. CRP can be measured in serum and is commonly used in epidemiological research as an indicator of inflammatory response to chronic stress [59].

IGF-1 is a hormone secreted by the liver and other cell types and can be measured in serum. It is of great importance for growth during childhood and puberty and has an anabolic function. It has been shown that glucocorticoids have a catabolic function [60] and that increased circulating glucocorticoids lead to inadequate anabolic growth hormone synthesis; when growth hormone is reduced, so is IGF-1 [61]. This reduction results in a catabolic/anabolic imbalance [62]. The literature search for this paper has not yielded any studies relating to IGF-1 levels and chronic stress in adolescents; however, studies on adults have shown that when exposed to chronic stress, IGF-1 levels decrease [63]. Primary biomarkers of chronic stress, biological tissues for their measurement, and their main functions are presented in Table 1.

\section{Secondary Outcomes of Stress}

Apart from the primary biomarkers of stress, secondary outcomes can also be taken into consideration when assessing the effect of chronic stress on adolescents. Secondary outcomes include anthropometric parameters, as well as metabolic and cardiovascular measures.

BMI, central adiposity, body size and form are all affected by chronic stress exposure. Persistent secretion of hormones related to stress during adolescence may pro- voke insulin or leptin resistance, which leads to fat storage [64]. High BMI has been associated with various chronic psychosocial stressors and has been linked to elevated saliva cortisol in adolescents. Chronic HPA axis activation accompanied by persistent glucocorticoid release leads to elevated insulin; this alteration in insulin levels can induce additional secondary changes in circulating cholesterol, $\alpha$-amylase, leptin, and glucose that can be useful outcome parameters when studying stress [65]. Furthermore, specific neurological measures could supplement the measurement of stress levels; it has been documented that chronic stress exposure is linked to decreased hippocampal volume [66]. Research has shown that smaller hippocampal volume is related to parental depressive symptomatology [67] and lower hippocampal activity has been related to post-traumatic symptoms in adolescents [68]. Under stress, cardiovascular tone and cytokine secretion are increased. Therefore, secondary markers related to the cardiovascular system, such as systolic and diastolic blood pressure, and heart rate or heart rate variability, are indicative of the effect of chronic stress on adolescents [69]. Studies on adolescents have demonstrated a correlation of increased blood pressure with stressful life events [70], whereas low heart rate variability has been linked to experiences of abuse [71].

\section{Psychometric Instruments of Adolescent Stress}

Weighted questionnaires and self-report instruments have profoundly affected scientific research on stress during all life stages. They have been used to measure stress 
either as the main outcome or as a predictive variable of outcomes, such as mental or physical disease [72]. Several psychometric instruments addressing adolescent stress have been developed.

Adolescent Life Change Event Scale was one of the first instruments to have been developed with a purpose of measuring adolescent stress. It describes 31 personal, social, and family changes which can potentially induce stress. Respondents are asked to rate each change on a scale from 1 to 5 indicating how upsetting this change would be for them. The instrument can be used to investigate correlations of stress with any physical or mental health issue in adolescents. However, the initial validation involved only a few more than 200 adolescents and has not been validated in other languages apart from English [73].

Adolescent Perceived Events Scale (APES) is a self-report questionnaire with 90 items, addressing issues that quite often affect adolescents. Each item describes a (positive or negative) stressful event, and in case the respondents have experienced the event within the past 6 months they are asked to rate the perceived desirability on a 4 -point scale. The scale was initially weighted solely in middle-class Caucasians, excluding other sociocultural groups, and has been validated only in the English and Spanish languages [74].

Urban Adolescent Life Experiences Scale was structured based on the Adolescent Perceived Events Scale, and examines chronic and episodic stress, along with major life events and daily hassles across four dimensions: (1) school, (2) family/social environment, (3) peer relations, and (4) personal relations. Adolescents are asked to declare how often they have been exposed to each of the stressors listed, on a 5-point scale. Again, the disadvantage of this scale is that it was weighted solely in African American youth [75].

Adolescent Stress Questionnaire consists of 58 items evaluating 10 different situations, able to induce stress: (1) family living conditions, (2) academic performance, (3) school attendance, (4) romantic relationships, (5) peer pressure, (6) teacher interaction, (7) uncertainty regarding the future, (8) conflict within the school/leisure environment, (9) financial pressure, and (10) emerging adult responsibility. Responses are given on a 5-point Likert scale and sub-scores for each dimension, as well as the total score derived from the sum of all responses [76]. The instrument has been translated and validated in several languages (i.e., Greek, Spanish, Dutch, Turkish, Norwegian, Hungarian, etc.) and is the primary questionnaire of choice for researchers when measuring adolescent stress

Measuring Adolescent Stress
[77]. A shorter version of the instrument has also been developed; however, it has not been validated yet in many languages apart from English, Greek, and Dutch [78].

\section{Factors to Consider when Measuring Stress in Adolescents}

Besides the ethical issues that must be carefully taken under consideration when involving minors in research studies, there are specific matters that should be addressed when measuring stress biomarkers during adolescence. As mentioned above, there are various ways in measuring biomarkers. The choice of the researchers should be based on the method that will provide the most accurate results and will not excessively burden the subjects involved in the study. For example, less invasive methods for cortisol assessment (hair, nail, saliva, or urine samples) are considered easier for the recruitment of participants. The method of analysis is an additional issue that needs to be carefully addressed; selecting the proper method of analysis could be a challenging subject, as some of the measurements lack a gold standard and for some, like cortisol, an established range varies excessively [79].

Researchers should also take into consideration the sex of the participants, as research studies on stress physiology and pathophysiology have reported some sexual dimorphism. Consequently, differences in primary stress biomarkers could also result in differences in secondary outcomes such as arterial blood pressure [80]. In addition, taking into consideration the fight-or-flight response, researchers have suggested that males and females respond differently, with males appearing as more aggressive (fight), and females tending to "flee" (flight) or seek help from their support system [81]. Researchers of adolescent stress should also always consider the pubertal status of their participants. Although it is not yet certain which mechanisms mediate the changes in the HPA axis function during adolescence, sex hormones (testosterone, estradiol, and progesterone) have appeared to affect the secretion of hormones such as the corticotropin-releasing hormone [82].

When using self-report instruments, researchers should always keep in mind the fact that recall bias is lurking, and thus, they cannot ground their results solely on such measurements. On the other hand, biomarkers are objective criteria; however, they vary within and between individuals. Also, in contrast to other specific pathological conditions, which result in specific biological respons-

Horm Res Paediatr 2023;96:74-82 DOI: $10.1159 / 000522387$ 
es affecting the system they relate to, stress affects several systems which can trigger or be triggered by additional pathologies. Therefore, it is wise to always interpret findings in the context of other co-existing pathologies. Lastly, but equally important, quantifying stress with the use of single biomarkers could be characterized as unrealistic. A set of biomarkers can provide a clearer picture of the effect of stress on different systems when the exposure is chronic.

\section{Conclusion}

Stress and adolescence are interwoven due to the inevitable physiological and psychosocial changes that occur during this stage of life. Considering that external stressors, such as academic performance or family and peer conflict, can be added to the expected stressors, measuring the response to stress is of great importance. Stress, differentiating itself from other pathologies, initiates a "non-specific" reaction, which involves several systems. To objectively evaluate stress, it is important for the researcher to be familiar with the condition under examination and with the appropriate corresponding stress indi- ces. Adequate evaluation of adolescents with selection of proper psychometric tests and biological markers can help design targeted interventions aiming to prevent or reverse the effects of physical and mental stressors that occur during adolescence, effects that can be carried into adulthood with detrimental consequences.

\section{Conflict of Interest Statement}

The authors have no conflicts of interest to declare.

\section{Funding Sources}

This research did not receive any specific grant from funding agencies in the public, commercial, or not-for-profit sectors.

\section{Author Contributions}

Ioulia Kokka: investigation and writing - original draft; George P. Chrousos and Christina Darviri: writing - review and editing; Flora Bacopoulou: writing - review and editing; visualization; project administration; and supervision.

\section{References}

1 Kokka I, Mourikis I, Nicolaides NC, Darviri C, Chrousos GP, Kanaka-Gantenbein C, et al. Exploring the effects of problematic internet use on adolescent sleep: a systematic review. Int J Environ Res Public Health. 2021 Jan 18; 18(2):760

2 Zhai ZW, Kirisci L, Tarter RE, Ridenour TA. Psychological dysregulation during adolescence mediates the association of parentchild attachment in childhood and substance use disorder in adulthood. Am J Drug Alcohol Abuse. 2014 Jan 1;40(1):67-74.

3 Farrell AK, Simpson JA, Carlson EA, Englund MM, Sung S. The impact of stress at different life stages on physical health and the buffering effects of maternal sensitivity. Health Psychol. 2017 Jan;36(1):35-44.

4 Green BN, Johnson CD, Adams A. Writing narrative literature reviews for peer-reviewed journals: secrets of the trade. J Chiropr Med. 2006 Sep;5(3):101-17.

5 Pare G, Kitsioy S. Methods for literature reviews. In: Lau F, Kuziemsky C, editors. Handbook of eHealth evaluation: an evidence-based approach. Victoria, BC: University of Victoria; 2017. Available from: https: //www.ncbi.nlm.nih.gov/books/ NBK481583/.

6 Berjot S, Gillet N. Stress and coping with discrimination and stigmatization. Front Psy- chol. 2011 [cited 2022 Jan 2];2:33. Available from: http: //journal.frontiersin.org/article/10.3389/fpsyg.2011.00033/abstract.

7 McEwen BS. Physiology and neurobiology of stress and adaptation: central role of the brain. Physiol Rev. 2007 Jul;87(3):873-904.

8 Bienertova Vasku J, Lenart P, Scheringer M. Eustress and distress: neither good nor bad, but rather the same? BioEssays. 2020 Jul; 42(7): 1900238.

9 Goldstein DS. Adrenal responses to stress. Cell Mol Neurobiol. 2010 Nov;30(8):143340.

10 Eiland L, Romeo RD. Stress and the developing adolescent brain. Neuroscience. 2013 Sep; 249:162-71.

11 Tsigos C, Kyrou I, Kassi E, Chrousos G. Stress: endocrine physiology and pathophysiology. In: Endotext [Internet]. South Dartmouth, MA: MDText.com; 2020. Available from: https: //www.ncbi.nlm.nih.gov/books/ NBK278995/.

12 Committee on Psychosocial Aspects of Child and Family Health; Committee on Early Childhood, Adoption, and Dependent Care, and Section on Developmental and Behavioral Pediatrics; Garner AS, Shonkoff JP, Siegel BS, Dobbins MI, et al. Early childhood adversity, toxic stress, and the role of the pediatrician: translating developmental science into lifelong health. Pediatrics. 2012 Jan 1;129(1): e224-31.

13 Tan S, Yip A. Hans Selye (1907-1982): founder of the stress theory. Singapore Med J. 2018 Apr;59(4):170-1.

14 Sen Y, Aygun D, Yilmaz E, Ayar A. Children and adolescents with obesity and the metabolic syndrome have high circulating cortisol levels. Neuro Endocrinol Lett. 2008 Feb; 29(1):141-5.

15 Lai JCL, Lee DYH, Leung MOY, Lam YW. Daily hassles, loneliness, and diurnal salivary cortisol in emerging adults. Horm Behav. 2019 Sep;115:104558.

16 van Andel HW, Jansen LM, Grietens H, Knorth EJ, van der Gaag RJ. Salivary cortisol: a possible biomarker in evaluating stress and effects of interventions in young foster children? Eur Child Adolesc Psychiatry. 2014 Jan; 23(1):3-12.

17 Bunea IM, Szentágotai-Tătar A, Miu AC. Early-life adversity and cortisol response to social stress: a meta-analysis. Transl Psychiatry. 2017 Dec;7(12):1274.

18 Adam EK, Quinn ME, Tavernier R, McQuillan MT, Dahlke KA, Gilbert KE. Diurnal cortisol slopes and mental and physical health outcomes: a systematic review and meta-analysis. Psychoneuroendocrinology. 2017 Sep; 83:25-41. 
19 Yonekura T, Takeda K, Shetty V, Yamaguchi M. Relationship between salivary cortisol and depression in adolescent survivors of a major natural disaster. J Physiol Sci. 2014 Jul;64(4): 261-7.

20 Martel FL, Hayward C, Lyons DM, Sanborn K, Varady S, Schatzberg AF. Salivary cortisol levels in socially phobic adolescent girls. Depress Anxiety. 1999;10(1):25-7.

21 Östberg V, Låftman SB, Modin B, Lindfors P. Bullying as a stressor in mid-adolescent girls and boys-associations with perceived stress, recurrent pain, and salivary cortisol. Int J Environ Res Public Health. 2018 Feb 20;15(2): 364.

22 Zhang J, Lam S-P, Kong AP, Ma RC, Li SX, Chan JW, et al. Family conflict and lower morning cortisol in adolescents and adults: modulation of puberty. Sci Rep. 2016 Apr; 6(1):22531

23 Dockray S, Susman EJ, Dorn LD. Depression, cortisol reactivity, and obesity in childhood and adolescence. J Adolesc Health. 2009 Oct; 45(4):344-50.

24 Nieman LK. Cushing's syndrome: update on signs, symptoms and biochemical screening. Eur J Endocrinol. 2015 Oct;173(4):M33-8.

25 Nieman LK. Diagnosis of cushing's syndrome in the modern era. Endocrinol Metab Clin North Am. 2018 Jun;47(2):259-73.

26 Antonelli G, Artusi C, Marinova M, Brugnolo L, Zaninotto M, Scaroni C, et al. Cortisol and cortisone ratio in urine: LC-MS/MS method validation and preliminary clinical application. Clin Chem Lab Med CCLM. 2014 Jan 1 [cited 2022 Jan 2];52(2):213-20. Available from: https: //www.degruyter.com/document/doi/10.1515/cclm-2013-0471/html.

27 Psarraki EE, Kokka I, Bacopoulou F, Chrousos GP, Artemiadis A, Darviri C. Is there a relation between major depression and hair cortisol? A systematic review and meta-analysis. Psychoneuroendocrinology. $2021 \mathrm{Feb}$ 124:105098.

28 de Azeredo LA, Viola TW, Rothmann LM, Trentin R, Arteche AX, Kristensen CH, et al. Hair cortisol levels and mental health problems in children and adolescents exposed to victimization. Stress. 2020 Sep 2;23(5):54655.

29 Sierau S, Glaesmer H, Klucken T, Stalder T. Hair cortisol, lifetime traumatic experiences and psychopathology in unaccompanied refugee minors. Psychoneuroendocrinology. 2019 Jun;104:191-4.

30 Genitsaridi SM, Karampatsou S, Papageorgiou I, Mantzou A, Papathanasiou C, Kassari $\mathrm{P}$, et al. Hair cortisol concentrations in overweight and obese children and adolescents. Horm Res Paediatr. 2019;92(4):229-36.

31 Kornelsen E, Buchan MC, Gonzalez A, Ferro MA. Hair cortisol concentration and mental disorder in children with chronic physical illness. Chronic Stress. 2019 Jan; 3 : 2470547019875116

32 Phillips R, Kraeuter AK, McDermott B, Lupien S, Sarnyai Z. Human nail cortisol as a retrospective biomarker of chronic stress: a systematic review. Psychoneuroendocrinology. $2021 \mathrm{Jan} ; 123: 104903$.

33 Doan SN, DeYoung G, Fuller-Rowell TE, Liu $\mathrm{C}$, Meyer J. Investigating relations among stress, sleep and nail cortisol and DHEA. Stress. 2018 Mar 4;21(2):188-93.

34 Charmandari E, Tsigos C, Chrousos G. Endocrinology of the stress response. Annu Rev Physiol. 2005 Mar 17;67(1):259-84.

35 Krahenbuhl GS. Adrenaline, arousal and sport. J Sports Med. 1975 May;3(3):117-21.

36 Kim N, Hughes TL, Park CG, Quinn L, Kong ID. Resting-state peripheral catecholamine and anxiety levels in korean male adolescents with internet game addiction. Cyberpsychol Behav Soc Netw. 2016 Mar;19(3):202-8.

37 Qi Z, Ding S. Obesity-associated sympathetic overactivity in children and adolescents: the role of catecholamine resistance in lipid metabolism. J Pediatr Endocrinol Metab. 2016 Jan 1 [cited 2022 Jan 2];29(2):113. Available from:

38 Graf WD, Unis AS, Yates CM, Sulzbacher S, Dinulos MB, Jack RM, et al. Catecholamines in patients with $22 \mathrm{q} 11.2$ deletion syndrome and the low-activity COMT polymorphism. Neurology. 2001 Aug 14;57(3):410-6.

39 Belujon P, Grace AA. Regulation of dopamine system responsivity and its adaptive and pathological response to stress. Proc Biol Sci. 2015 Apr 22;282(1805):20142516.

40 Paus T, Keshavan M, Giedd JN. Why do many psychiatric disorders emerge during adolescence? Nat Rev Neurosci. 2008 Dec;9(12): 947-57.

41 Dutheil F, de Saint Vincent S, Pereira B, Schmidt J, Moustafa F, Charkhabi M, et al. DHEA as a biomarker of stress: a systematic review and meta-analysis. Front Psychiatry. 2021 Jul 6;12:688367.

42 van Niekerk JK, Huppert FA, Herbert J. Salivary cortisol and DHEA: association with measures of cognition and well-being in normal older men, and effects of three months of DHEA supplementation. Psychoneuroendocrinology. 2001 Aug;26(6):591-612.

43 Ceresini G, Morganti S, Rebecchi I, Freddi M, Ceda GP, Banchini A, et al. Evaluation of the circadian profiles of serum dehydroepiandrosterone (DHEA), cortisol, and cortisol/ DHEA molar ratio after a single oral administration of DHEA in elderly subjects. Metabolism. 2000 Apr;49(4):548-51.

44 King LS, Graber MG, Colich NL, Gotlib IH. Associations of waking cortisol with DHEA and testosterone across the pubertal transition: effects of threat-related early life stress. Psychoneuroendocrinology. 2020 May; 115: 104651.

45 Marceau K, Shirtcliff EA, Hastings PD, Klimes-Dougan B, Zahn-Waxler C, Dorn LD, et al. Within-adolescent coupled changes in cortisol with DHEA and testosterone in response to three stressors during adolescence. Psychoneuroendocrinology. 2014 Mar;41: 33-45.
46 Zhang JM, An J. Cytokines, inflammation, and pain. Int Anesthesiol Clin. 2007;45(2): 27-37.

47 Morey JN, Boggero IA, Scott AB, Segerstrom SC. Current directions in stress and human immune function. Curr Opin Psychol. 2015 Oct; 5:13-7.

48 Miller GE, Chen E, Fok AK, Walker H, Lim A, Nicholls EF, et al. Low early-life social class leaves a biological residue manifested by decreased glucocorticoid and increased proinflammatory signaling. Proc Natl Acad Sci. 2009 Aug 25;106(34):14716-21.

49 Allen JP, Loeb EL, Tan JS, Narr RK, Uchino $\mathrm{BN}$. The body remembers: adolescent conflict struggles predict adult interleukin-6 levels. Dev Psychopathol. 2018 Oct;30(4):143545.

50 Caserta MT, Wyman PA, Wang H, Moynihan J, O'Connor TG. Associations among depression, perceived self-efficacy, and immune function and health in preadolescent children. Dev Psychopathol. 2011 Nov;23(4): 1139-47.

51 Sutton CE, Lalor SJ, Sweeney CM, Brereton CF, Lavelle EC, Mills KH. Interleukin-1 and IL-23 induce innate IL-17 production from gammadelta $\mathrm{T}$ cells, amplifying Th17 responses and autoimmunity. Immunity. 2009 Aug;31(2):331-41.

52 Bernton EW, Beach JE, Holaday JW, Smallridge RC, Fein HG. Release of multiple hormones by a direct action of interleukin-1 on pituitary cells. Science. 1987 Oct 23;238(4826): $519-21$.

53 Berkenbosch F, van Oers J, del Rey A, Tilders F, Besedovsky H. Corticotropin-releasing factor-producing neurons in the rat activated by interleukin-1. Science. 1987 Oct 23;238(4826) 524-6.

54 Amaral GA, Alves JD, Honorio-França AC, Fagundes DL, Araujo GG, Lobato NS, et al. Interleukin 1-beta is linked to chronic lowgrade inflammation and cardiovascular risk factors in overweight adolescents. Endocr Metab Immune Disord Drug Targets. 2020 Jul 17;20(6):887-94.

55 Idriss HT, Naismith JH. TNF alpha and the TNF receptor superfamily: structure-function relationship(s). Microsc Res Tech. 2000 Aug 1;50(3):184-95.

56 Dixon D, Meng H, Goldberg R, Schneiderman N, Delamater A. Stress and body mass index each contributes independently to tumor necrosis factor-alpha production in prepubescent Latino children. J Pediatr Nurs. 2009 Oct;24(5):378-88.

57 Thompson D, Pepys MB, Wood SP. The physiological structure of human C-reactive protein and its complex with phosphocholine. Structure. 1999 Feb;7(2):169-77.

58 Fuligni AJ, Telzer EH, Bower J, Cole SW, Kiang L, Irwin MR. A preliminary study of daily interpersonal stress and C-reactive protein levels among adolescents from Latin American and European backgrounds. Psychosom Med. 2009 Apr;71(3):329-33. 
59 Häfner S, Emeny RT, Lacruz ME, Baumert J, Herder C, Koenig W, et al. Association between social isolation and inflammatory markers in depressed and non-depressed individuals: results from the MONICA/KORA study. Brain Behav Immun. 2011 Nov;25(8): 1701-7.

60 Asensio C, Muzzin P, Rohner-Jeanrenaud F. Role of glucocorticoids in the physiopathology of excessive fat deposition and insulin resistance. Int J Obes Relat Metab Disord. 2004 Dec;28 Suppl 4(S4):S45-52.

61 Malarkey WB, Wu H, Cacioppo JT, Malarkey KL, Poehlmann KM, Glaser R, et al. Chronic stress down-regulates growth hormone gene expression in peripheral blood mononuclear cells of older adults. Endocrine. 1996 Aug; 5(1):33-9.

62 Epel E, Adler N, Ickovics J, McEwen B. Social status, anabolic activity, and fat distribution. Ann N Y Acad Sci. 1999 Dec;896(1):424-6.

63 Bernton E, Hoover D, Galloway R, Popp K. Adaptation to chronic stress in military trainees. Adrenal androgens, testosterone, glucocorticoids, IGF-1, and immune function. Ann N Y Acad Sci. 1995 Dec 29;774:217-31.

64 Pervanidou P, Chrousos GP. Metabolic consequences of stress during childhood and adolescence. Metabolism. 2012 May;61(5):6119.

65 Ruttle PL, Klein MH, Slattery MJ, Kalin NH, Armstrong JM, Essex MJ. Adolescent adrenocortical activity and adiposity: differences by sex and exposure to early maternal depression. Psychoneuroendocrinology. 2014 Sep; 47:68-77.

66 Tottenham N. A review of adversity, the amygdala and the hippocampus: a consideration of developmental timing. Front Hum Neurosci. 2009 [cited 2022 Jan 2];3:68.
67 Rao U, Chen LA, Bidesi AS, Shad MU, Thomas MA, Hammen CL. Hippocampal changes associated with early-life adversity and vulnerability to depression. Biol Psychiatry. 2010 Feb;67(4):357-64.

68 Carrion VG, Haas BW, Garrett A, Song S, Reiss AL. Reduced hippocampal activity in youth with posttraumatic stress symptoms: an fMRI Study. J Pediatr Psychol. 2010 Jun 1; 35(5):559-69.

69 Steptoe A, KivimÃaki M. Stress and cardiovascular disease: an update on current knowledge. Annu Rev Public Health. 2013 Mar 18; 34(1):337-54.

70 Brady SS, Matthews KA. Chronic stress influences ambulatory blood pressure in adolescents. Ann Behav Med. 2006 Feb;31(1):80-8.

71 Murali R, Chen E. Exposure to violence and cardiovascular and neuroendocrine measures in adolescents. Ann Behav Med. 2005 Oct 1; 30(2):155-63.

72 Strodl E, Kenardy J, Aroney C. Perceived stress as a predictor of the self-reported new diagnosis of symptomatic CHD In older women. Int J Behav Med. 2003 Sep;10(3): 205-20.

73 Yeaworth RC, York J, Hussey MA, Ingle ME, Goodwin T. The development of an adolescent life change event scale. Adolescence. 1980;15(57):91-7.

74 Compas BE, Davis GE, Forsythe CJ, Wagner $\mathrm{BM}$. Assessment of major and daily stressful events during adolescence: the adolescent perceived events scale. J Consult Clin Psychol. 1987 Aug;55(4):534-41.
75 Allison KW, Burton L, Marshall S, Perez-Febles A, Yarrington J, Kirsh LB, et al. Life experiences among urban adolescents: examining the role of context. Child Dev. 1999 Jul;70(4): 1017-29.

76 Byrne DG, Davenport SC, Mazanov J. Profiles of adolescent stress: the development of the adolescent stress questionnaire (ASQ). J Adolesc. 2007 Jun;30(3):393-416.

77 Ertanir B, Rietz C, Graf U, Kassis W. A crossnational validation of the shortened version of the adolescent stress questionnaire (ASQS) among adolescents from Switzerland, Germany, and Greece. Front Psychol. 2021 Apr 9; 12:619493.

78 McKay M, Andretta J, Perry J. The shortened version of the adolescent stress questionnaire (ASQ-S; Sweden): a validation study in United Kingdom adolescents. Scand J Child Adolesc Psychiatry Psychol. 2019;7:81-7.

79 Russell E, Koren G, Rieder M, Van Uum S. Hair cortisol as a biological marker of chronic stress: current status, future directions and unanswered questions. Psychoneuroendocrinology. 2012 May;37(5):589-601.

80 Wener MH, Daum PR, McQuillan GM. The influence of age, sex, and race on the upper reference limit of serum C-reactive protein concentration. J Rheumatol. 2000 Oct;27(10): 2351-9.

81 Taylor SE, Klein LC, Lewis BP, Gruenewald TL, Gurung RA, Updegraff JA. Biobehavioral responses to stress in females: tend-and-befriend, not fight-or-flight. Psychol Rev. 2000; 107(3):411-29.

82 Klein ZA, Romeo RD. Changes in hypothalamic-pituitary-adrenal stress responsiveness before and after puberty in rats. Horm Behav. 2013 Jul;64(2):357-63. 